\title{
On the emergence of molecular structure
}

\author{
Matyus, E ; Hutter, J ; Mueller-Herold, U ; Reiher, M
}

\begin{abstract}
The structure of $\mathrm{a}(+/-), \mathrm{a}(+/-), \mathrm{b}(+/-)$-type Coulombic systems is characterized by the effective ground-state density of the a-type particles, computed via nonrelativistic quantum mechanics without introduction of the Born-Oppenheimer approximation. A structural transition is observed when varying the relative mass of the a- and b-type particles, e.g., between atomic $\mathrm{H}-$ and molecular $\mathrm{H}-2(+)$. The particle-density profile indicates a molecular-type behavior for the positronium ion, $\operatorname{Ps}(-)$.
\end{abstract}

DOI: https://doi.org/10.1103/PhysRevA.83.052512

Posted at the Zurich Open Repository and Archive, University of Zurich

ZORA URL: https://doi.org/10.5167/uzh-48896

Journal Article

Accepted Version

Originally published at:

Matyus, E; Hutter, J; Mueller-Herold, U; Reiher, M (2011). On the emergence of molecular structure. Physical Review A, 83(5):082512.

DOI: https://doi.org/10.1103/PhysRevA.83.052512 


\title{
On the emergence of molecular structure
}

\author{
Edit Mátyus, ${ }^{1}$ Jürg Hutter, ${ }^{2}$ Ulrich Müller-Herold,${ }^{3}$ and Markus Reiher ${ }^{1}$ \\ ${ }^{1}$ Laboratory of Physical Chemistry, ETH Zürich, \\ Wolfgang-Pauli-Str. 10, CH-8093 Zürich, Switzerland \\ ${ }^{2}$ Institute of Physical Chemistry, University of Zürich, \\ Winterthurerstrasse 190, CH-8057 Zürich, Switzerland \\ ${ }^{3}$ Institute of Biogeochemistry and Pollutant Dynamics, \\ ETH Zürich, Universitätstrasse 16, CH-8092 Zürich, Switzerland
}

(Dated: March 17, 2011)

\begin{abstract}
The structure of $\left\{a^{ \pm}, a^{ \pm}, b^{\mp}\right\}$-type Coulombic systems is characterized by the effective ground-state density of the $a$-type particles, computed via non-relativistic quantum mechanics without the introduction of the Born-Oppenheimer approximation. A structural transition is observed when varying the relative mass of the $a$ - and $b$-type particles, e.g., between atomic $\mathrm{H}^{-}$and molecular $\mathrm{H}_{2}^{+}$. The particle-density profile indicates a moleculartype behavior for the positronium ion, $\mathrm{Ps}^{-}$.
\end{abstract}




\section{INTRODUCTION}

Stability regions of few-particle systems in terms of the physical parameters of non-relativistic quantum mechanics have been studied extensively [1-5] and provide useful pieces of information for studying matter-antimatter [6-10] interactions and exotic assemblies of particles [5]. Special structural regions of stable assemblies of electrons and nuclei, especially those corresponding to a molecular structure, are of particular interest for molecular physics and chemistry.

Molecular structure is traditionally interpreted within the Born-Oppenheimer (BO) approximation [11-14]. The BO or clamped-nuclei approximation leads to the introduction of the potential energy surfaces (PESs) as well as to the concept of equilibrium structures defined as local minima of the PES for various molecular systems. Whether equilibrium or rovibrationally averaged effective structures [15] are used to characterize the structure of molecules [16], the description relies on the BO approximation. Thus, the BO approximation provides mathematical basis for several important notions central to the present-day thinking about molecular systems. Nevertheless, it is not without conceptual problems. As Primas explains [17]: "We describe the six degrees of freedom of the ground state of the helium atom (considered as 3-particle problem with the center-of-mass motion separated) as a problem of two interacting particles in an external Coulomb potential. However, in the case of the molecule $\mathrm{H}_{2}^{+}$we discuss the very same type of differential equation in an entirely different way, and split the 6 degrees of freedom into 1 vibrational mode, 2 rotational modes, and 3 electronic type degrees of freedom. This qualitatively different description does by no means follow from a purely mathematical discussion." This reasoning indicates that the introduction of the $\mathrm{BO}$ approximation, by itself, makes an a priori assumption about the existence of a particular structure for atoms and another one for molecules.

Then, the following question arises: is it possible to observe the emergence of molecular structure as an inherent feature of non-relativistic quantum theory for certain ranges of its parameters without making empirical assumptions? Once stability regions of few-particle systems were discovered $[2,5]$, one makes a step forward, onto the next level of organization of matter, and wants to discover the special structural 
regions in terms of the physical parameters of the theory. To address this question, the simplest possible family of systems comprising both atomic- and molecular-type objects is studied in this work: three-particle systems with two identical particles of unit charges and a third one with opposite charge [18-23].

This system was studied by one of the present authors in the analytically solvable so-called $1 / r^{2}$ harmonium or Hooke-Calogero model [24] of a three-particle system $\{a, a, b\}$ where the attraction between $a$ and $b$ is harmonic and the repulsion between the two $a$-particles is given by an inverse square potential. The emergence of new structure was observed in the ground-state density $\rho_{a}(\boldsymbol{R}), \boldsymbol{R} \in \mathbb{R}^{3}$ of the $a$-particles. It turned out that for large mass of $b$ and small mass of $a \rho_{a}$ is a Gaussian-type function, centered at the origin, whereas for large mass of $a \rho_{a}$ is a positive spherical function with a minimum at the origin, corresponding to an isotropically rotating quantum mechanical dumbbell. At a critical value of the $a$-mass a topological transition of $\rho_{a}$ was observed between the two domains corresponding to the emergence of a molecular structure arising from an initially atom-like shape. Emergence through this kind of topological transition is clearly different from the more familiar variants where singular limits and symmetry breaking appear as the key formal ingredients $[25]$.

Does this type of analysis apply to "real-life" Coulomb interactions? The nonrelativistic quantum Hamiltonian of $\left\{a^{ \pm}, a^{ \pm}, b^{\mp}\right\}$-type three-particle systems in Hartree atomic units is

$$
\hat{H}\left(m_{a}, m_{b}, \boldsymbol{x}\right)=-\frac{1}{2 m_{a}} \Delta_{\boldsymbol{x}_{1}}-\frac{1}{2 m_{a}} \Delta_{\boldsymbol{x}_{2}}-\frac{1}{2 m_{b}} \Delta_{\boldsymbol{x}_{3}}+\frac{1}{x_{12}}-\frac{1}{x_{13}}-\frac{1}{x_{23}},
$$

where $m_{a}$ and $m_{b}$ are the masses assigned to the particles. As proven in Refs. [2] and [4], these systems are stable over the whole mass range.

A special property of the Coulomb Hamiltonian is its mass-scale similarity [2]:

$$
\hat{H}\left(\eta m_{a}, \eta m_{b}, \boldsymbol{x}\right)=\eta \hat{H}\left(m_{a}, m_{b}, \eta \boldsymbol{x}\right),
$$

$\forall \eta \in \mathbb{R} \backslash\{0\}$. This means that scaling the masses by a factor $\eta$ is equivalent to scaling the energy and shrinking the length by the factor $\eta$. The important consequence of the "mass-scaling rule" for this work is that it is sufficient to consider the 
ratio of the masses, $m_{a} / m_{b}$. Additionally, the charge inversion invariance, indicated in the notation $\left\{a^{ \pm}, a^{ \pm}, b^{\mp}\right\}$, is self-explanatory by inspecting the structure of the Coulomb Hamiltonian. It is worth noting that the mass-scale similarity is special to the Coulomb Hamiltonian, and does not apply to the $1 / r^{2}$ harmonium model, as discussed in Ref. [24].

\section{COMPUTATIONAL METHODS}

In order to compute the ground-state wave function of the Hamiltonian for various $m_{a}$ and $m_{b}$ values, a computer program based on a variational procedure was developed. In spite of the common practice in molecular physics and spectroscopy of introducing rotational and internal coordinates [26-31], for simplicity, the Hamiltonian was expressed here in terms of translationally invariant Cartesian coordinates and the translation of the center of mass was separated $[32,33]$. The matrix representation of the translationally invariant Hamiltonian was constructed using explicitly correlated Gaussian functions with zero orbital angular momentum and natural parity [34]. The nonlinear parameters of the basis functions were optimized using the stochastic variational method [32], based on earlier works [35, 36], during the course of the computation. The eigenfunctions obtained are space-inversion invariant, have zero orbital angular momentum, and are invariant under the permutation of the identical particles $a$. The resulting wave function meets the Pauli principle for instance for two spin-1/2 fermionic $a$-type particles with antiparallel spins.

\section{PARTICLE DENSITY}

As all particles are treated without introducing any approximate separation, in principle, the non-relativistic limit can be approached numerically [37-42]. At the same time, the structural analysis of the results might not be as straightforward $[13,43,44]$ as in the case of the BO treatment. In order to characterize the structure within all-particle, non-Born-Oppenheimer computations, particle-density-like quantities, such as the "pseudoparticle density" [45] and the "mass density" [24, 46] or correlation functions were evaluated [9]. 


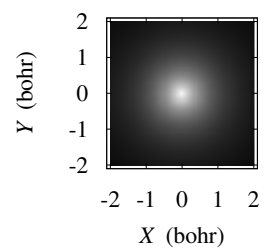

(a)

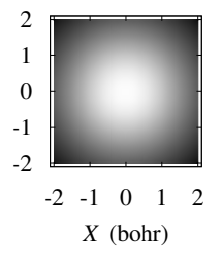

(b)

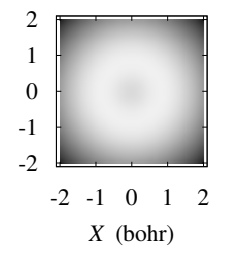

(c)

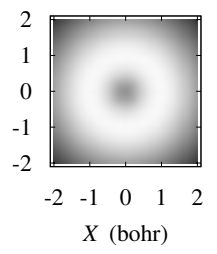

(d)

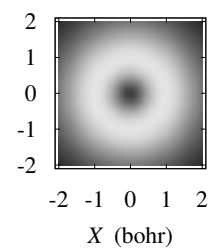

(e)

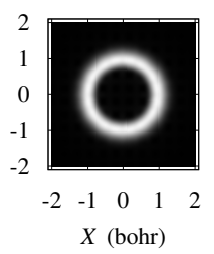

(f)

$$
\left\{1^{ \pm}, 1^{ \pm}, m_{\mathrm{p}}^{\mp}\right\} \quad\left\{1^{ \pm}, 1^{ \pm}, 2^{\mp}\right\} \quad\left\{1^{ \pm}, 1^{ \pm}, 1^{\mp}\right\} \quad\left\{2^{ \pm}, 2^{ \pm}, 1^{\mp}\right\} \quad\left\{5^{ \pm}, 5^{ \pm}, 1^{\mp}\right\} \quad\left\{m_{\mathrm{p}}^{ \pm}, m_{\mathrm{p}}^{ \pm}, 1^{\mp}\right\}
$$

FIG. 1: Transition from an atomic- to a molecular-type distribution of the $a$ particles in $\left\{a^{ \pm}, a^{ \pm}, b^{\mp}\right\}$-type Coulombic systems. In each graph the normalized density plot of $\rho_{a}(\boldsymbol{R})$ is shown for $\boldsymbol{R}=(X, Y, 0)$ in terms of $X, Y \in[-2,2]$ bohr. The assembled particles are specified as $\left\{m_{a}^{ \pm}, m_{a}^{ \pm}, m_{b}^{\mp}\right\}$, where $m_{a}$ and $m_{b}$ are the masses and $m_{\mathrm{p}}=1836.15267247$ [47] in units of the mass of an electron. The center of mass is the center of each plot.

In this work the distribution of the particles in the ground state, $\Psi_{0}$, is characterized by an effective particle density introduced in the spirit of the mass density in Ref. [24]. It is measured from the center of mass (CM) and is defined for the $a$ particles as

$$
\rho_{a}(\boldsymbol{R})=\left\langle\Psi_{0}(\boldsymbol{r})\left|\hat{\rho}_{a}(\boldsymbol{r}, \boldsymbol{R})\right| \Psi_{0}(\boldsymbol{r})\right\rangle, \quad \boldsymbol{R} \in \mathbb{R}^{3},
$$

where

$$
\hat{\rho}_{a}(\boldsymbol{r}, \boldsymbol{R})=\sum_{i=1}^{2} \delta\left(\boldsymbol{d}_{i}(\boldsymbol{r})-\boldsymbol{R}\right)
$$

and the displacement vectors $\boldsymbol{d}_{1}=\boldsymbol{x}_{1}-\boldsymbol{x}_{\mathrm{CM}}$ and $\boldsymbol{d}_{2}=\boldsymbol{x}_{2}-\boldsymbol{x}_{\mathrm{CM}}$ were written in terms of the translationally invariant Cartesian coordinates, $\boldsymbol{r}$.

\section{RESULTS AND DISCUSSION}

Due to the spherical symmetry of the ground state with zero orbital angular momentum $\rho_{a}$ is also spherically symmetric. Figures 1-3 present cuts of $\rho_{a}$ for various $m_{a}$ and $m_{b}$ values. According to the mass-scale similarity of the Coulomb Hamiltonian, systems with different $m_{a}$ and $m_{b}$ values but an identical $m_{a} / m_{b}$ ratio are similar in the sense of Eq. (2). Additionally, the charge inversion invariance also 
holds. Thus, in what follows numerical results are presented for assemblies of $a^{ \pm}$ and $b^{\mp}$ particles with opposite but in absolute value unit charges and for various $m_{a} / m_{b}$ ratios.

In Figures 1-2 cuts of the 3-dimensional, spherically symmetric particle density are shown to present the variation of $\rho_{a}$ in terms of $m_{a} / m_{b}$. In Figure 1 density plots of $\rho_{a}(\boldsymbol{R})$ along the $Z=0$ plane, i.e., for $\boldsymbol{R}=(X, Y, 0)$ within the interval $X, Y \in[-2,2]$ bohr are given. In Figure 2 the qualitative change of the profile of $\rho_{a}(\boldsymbol{R})$ along a ray, e.g., for the cut $\boldsymbol{R}=(X, 0,0)$ and $X \in[-10,10]$ bohr is shown in the neighborhood of the transition point.

For a small $m_{a} / m_{b}$ mass ratio $\rho_{a}$ has a maximum at the origin, which means that the distribution of the $a$-type particles is centered at the center of mass of the system. As $m_{a} / m_{b}$ increases the distribution becomes more and more diffuse until a local minimum shows up at the center of mass. This point can be interpreted as a transition point where the "atomic" and "molecular" structures coalesce and the atomic-type, center-of-mass centered distribution transforms to a molecular-type distribution with maxima off-centered from the center of mass. According to our computations this transition takes place within the interval $0.4<m_{a} / m_{b}<0.8$.

Then, by further increasing $m_{a} / m_{b}$, the localization of the $a$-type particles displaced from the center of mass becomes more and more pronounced, and the picture of a molecular system becomes evident. Although both the atomic and the molecular systems are sphere-like due to the ascribed symmetry, in the molecular case the $a$-type particles are most likely found in a shell at a given distance from the center of mass. This shell-like distribution reminds us of a rotating dumbbell in its quantum mechanical ground state, with zero rotational angular momentum. Furthermore, the finite width of the shell corresponds to the zero-point vibration of the nuclei in the BO picture. In line with this picture an efficient variational description of energy levels and properties of molecular systems without introducing the BO approximation requires the usage of basis functions mimicking this hollowed distribution of the heavy particles $[23,32,33]$.

As to the limiting cases, one may reason as follows. As $m_{a} / m_{b} \rightarrow 0$, the particle density becomes more and more pointed and at the limit it has a cusp at the center of mass, as it is known from electronic structure theory of molecules with clamped 


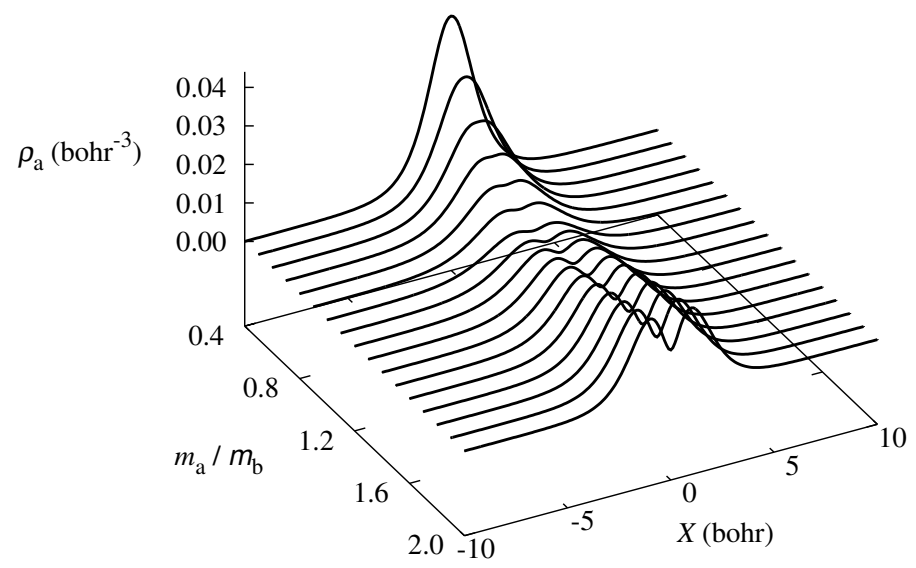

FIG. 2: Emergence of molecular structure in $\left\{a^{ \pm}, a^{ \pm}, b^{\mp}\right\}$-type Coulombic systems. $\rho_{a}(\boldsymbol{R})$ is shown along the ray $\boldsymbol{R}=(X, 0,0)$ and $X \in[-10,10]$ bohr for ratios $m_{a} / m_{b} \in[0.4,2.0]$. For $m_{a} / m_{b} \leq 1: m_{a}=1$ and for $m_{a} / m_{b}>1: m_{b}=1$ were fixed measured in units of the mass of an electron. Note the mass-scale similarity of Coulombic systems.

nuclei $[11,48]$. At the same time, as $m_{a} / m_{b} \rightarrow+\infty$, the shell of the $a$-type particles becomes infinitely thin. In the intermediate cases, $\rho_{a}$, as defined in Eqs. (3) and (4), has no cusps at the maxima, as in a qualitative picture, it is smeared due to the motion of the third particle.

As in the variational computations no a priori assumptions were made about the distribution of the particles, the atomic to molecular transition appears solely as a special feature of the theory for certain ranges of its parameters. Although one might note that there is a compositional asymmetry in the assembly of two identical particles and a third, single, different one, for the symmetric $\left\{a^{ \pm}, a^{ \pm}, b^{\mp}, b^{\mp}\right\}$-type systems the $m_{a} / m_{b} \rightarrow+\infty$ and $m_{b} / m_{a} \rightarrow+\infty$ limits are identical due to the massscale similarity and the charge-inversion symmetry of the Coulomb Hamiltonian.

While in Figure 2 the qualitative change of the particle density was presented as a function of the $m_{a} / m_{b}$ ratio, in Figure 3 three physically realizable sections of the surface are shown: the atomic-like $\mathrm{H}^{-}=\left\{\mathrm{e}^{-}, \mathrm{e}^{-}, \mathrm{p}^{+}\right\}$, the $\mathrm{Ps}^{-}=\left\{\mathrm{e}^{-}, \mathrm{e}^{-}, \mathrm{e}^{+}\right\}$, and the molecular-like $\mathrm{H}_{2}^{+}=\left\{\mathrm{p}^{+}, \mathrm{p}^{+}, \mathrm{e}^{-}\right\}$(note the mass-scale similarity and the chargeinversion invariance of the Coulomb Hamiltonian). The transition from the atomic, $\mathrm{H}^{-}$, to the molecular, $\mathrm{H}_{2}^{+}$, particle density profile can not be interpreted within the 


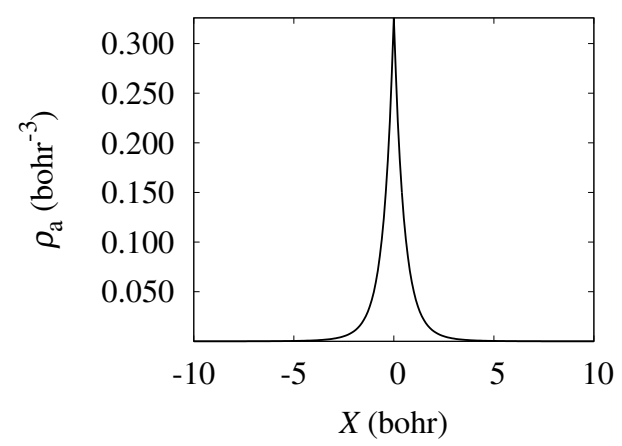

(a) $m_{a} / m_{b}=m_{\mathrm{e}} / m_{\mathrm{p}} \Rightarrow\left\{\mathrm{e}^{-}, \mathrm{e}^{-}, \mathrm{p}^{+}\right\}=\mathrm{H}^{-}$

"atomic-type" distribution

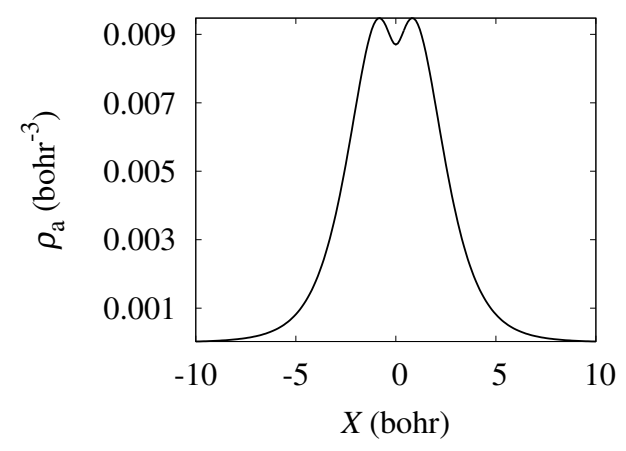

(b) $m_{a} / m_{b}=1 / 1 \Rightarrow\left\{\mathrm{e}^{-}, \mathrm{e}^{-}, \mathrm{e}^{+}\right\}=\mathrm{Ps}^{-}$

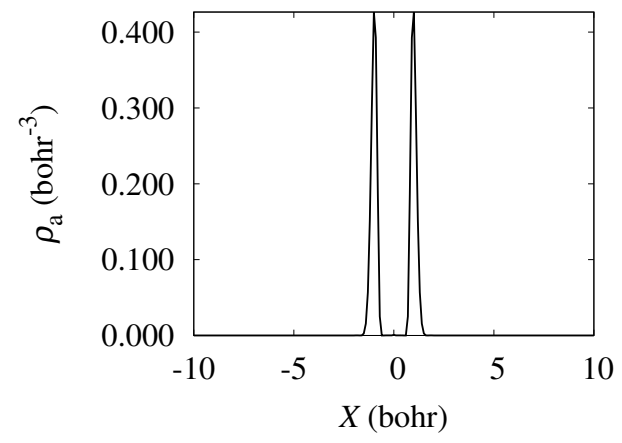

(c) $m_{a} / m_{b}=m_{\mathrm{p}} / m_{\mathrm{e}} \Rightarrow\left\{\mathrm{p}^{+}, \mathrm{p}^{+}, \mathrm{e}^{-}\right\}=\mathrm{H}_{2}^{+}$

"molecular-type" distribution

FIG. 3: $a$-particle density profile in $\left\{a^{ \pm}, a^{ \pm}, b^{\mp}\right\}$-type Coulombic systems. $\rho_{a}(\boldsymbol{R})$ is plotted along the ray $\boldsymbol{R}=(X, 0,0)$ within the interval $X \in[-10,10]$ bohr. The origin coincides with the center of mass. $m_{\mathrm{p}} / m_{\mathrm{e}}=1836.15267247$ [47].

BO approximation, as it introduces two completely different descriptions, i.e., two different sets of differential equations to be solved, for the $\mathrm{H}^{-}$and the $\mathrm{H}_{2}^{+}$threeparticle systems [17]. 
Furthermore, everyone would agree that $\mathrm{H}^{-}$is an atomic-type, whereas $\mathrm{H}_{2}^{+}$is a molecular-type object in the ground state. Indeed, the particle density plots presented in Figure 3 are in good agreement with the properties of these well-known regimes of few-particle systems. It is interesting to consider if atomic- and moleculartype three-particle systems can be categorized according to the qualitative profile of $\rho_{a}$. If so, then according to this classification, which can be considered as a new "definition" of a molecule for three-particle systems, $\mathrm{Ps}^{-}$would also belong to the set of molecular-like systems even though one would not expect that it is tractable within the BO approximation. Interestingly, the resonance spectrum of $\mathrm{Ps}^{-}$could be understood based on an adiabatic treatment of the interelectronic distance and it was possible to carry out a systematic classification of its features using molecular-type quantum numbers similar to $\mathrm{H}_{2}^{+}$[6]. Furthermore, the analysis of the eigenspectrum of the non-relativistic Hamiltonian in terms of the particle charges and masses of three-particle systems allowed to study quantum critical phenomena and based on the position of the "pseudocritical points" $\mathrm{Ps}^{-}$was recognized as a molecular-like system [49]. In the present work, the particle distribution considered for ground states of three-particle systems provided a direct indication of the molecular-like structure of $\mathrm{Ps}^{-}$in agreement with the earlier works $[6,49]$.

These observations trigger the following question: Is it possible to introduce the notion of molecules and molecular structure without relying in some way on the Born-Oppenheimer approximation?

\section{CONCLUSIONS}

For the $a$ particles being electrons, connections between the topology of $\rho_{a}$ and various elements of molecular structure are known since long. In particular the formation and breaking of chemical bonds has been studied in great detail [50]. In the present approach for the first time topological changes were considered due to varying masses of the respective particles. For that purpose Coulombic systems with two identical particles with unit charges and a third, oppositely charged particle were considered within non-relativistic quantum mechanics and without making a priori assumptions on the structure, i.e., without introducing the Born-Oppenheimer 
approximation. The structure of these assemblies was analyzed by computing the effective ground-state particle density for the identical particles. As a "mass-scale similarity" applies for the Coulomb Hamiltonian, it was sufficient to compute the particle density for various mass ratios. If the mass ratio of the identical particles and the third particle is small, the density of the identical particles is centered at the center of mass, whereas by increasing the relative mass a qualitative change of the particle density takes place and its maxima appear off-centered from the center of mass. In topological terms this means a transition from a single attractor $(3,-3)$ at $R=0$ to a cage point $(3,3)$ together with a critical $(1,-1)$ sphere [51]. It corresponds to a changeover from atomic- to molecular-type density distribution, in line with the results in the Hooke-Calogero model [24].

As charge-inversion invariance also applies for the Coulomb Hamiltonian, one can invert the charges without changing the energy, the wave function or the particle density. Making use of this property, a continuous transition was presented from the atomic-like $\mathrm{H}^{-}=\left\{\mathrm{e}^{-}, \mathrm{e}^{-}, \mathrm{p}^{+}\right\}$ion to the molecular-like $\mathrm{H}_{2}^{+}=\left\{\mathrm{p}^{+}, \mathrm{p}^{+}, \mathrm{e}^{-}\right\}$ion by inspecting the particle density in terms of the relative masses. Hence, the emergence of molecular structure is witnessed as it results from the inherent nature of the physical theory for various ranges of its parameters without making empirical assumptions about the structure.

\section{Acknowledgments}

E. M. acknowledges support from the TH Grant O-20615-09. E. M. is thankful to Profs. Attila G. Császár and Edward F. Valeev for discussions on the non-BornOppenheimer theory.

[1] R. N. Hill, J. Math. Phys. 18, 2316 (1977).

[2] A. Martin, J.-M. Richard, and T. T. Wu, Phys. Rev. A 46, 3697 (1992).

[3] C. D. Lin, Phys. Rep. 257, 1 (1995).

[4] A. A. Farajian, K. Esfarjani, and Y. Kawazoe, J. Phys. B: At. Mol. Opt. Phys. 32, 749 (1999). 
[5] E. A. G. Armour, J.-M. Richard, and K. Varga, Phys. Rep. 413, 1 (2005).

[6] J. M. Rost and D. Wintgen, Phys. Rev. Lett. 69, 2499 (1992).

[7] A. P. Mills, Jr., Phys. Rev. Lett. 47, 717 (1981).

[8] J. Botero and C. H. Greene, Phys. Rev. Lett. 56, 1366 (1986); J. Botero, Phys. Rev. A 35, 36 (1987).

[9] K. Varga, J. Usukura, and Y. Suzuki, Phys. Rev. Lett. 80, 1876 (1999); J. Usukura, K. Varga, and Y. Suzuki, Phys. Rev. A 58, 1918 (1998).

[10] D. B. Cassidy and A. P. Mills, Jr., Nature 449, 195 (2007).

[11] M. Born and R. Oppenheimer, Ann. der Phys. 84, 457 (1927).

[12] B. T. Sutcliffe, Theor. Chem. Acc. 127, 121 (2010).

[13] B. T. Sutcliffe and R. G. Woolley, Phys. Chem. Chem. Phys. 7, 3667 (2005).

[14] G. A. Hagedorn and A. Joye, Mathematical Analysis of Born-Oppenheimer Approximations. Spectral Theory and Mathematical Physics: A Festschrift in Honor of Barry Simon's 60th Birthday. Part I: Quantum Field Theory, Statistical Mechanics, and Non-relativistic Quantum Systems, Ed. by F. Gesztesy, P. Deift, C. Galvez, P. Perry, and W. Schlag. Amer. Math. Soc. Proceedings of Symposia in Mathematics 76, Part 1, 203-226 (2007).

[15] G. Czakó, E. Mátyus, and A. G. Császár, J. Phys. Chem. A 113, 11665 (2009).

[16] Equilibrium Molecular Structures: From Spectroscopy to Quantum Chemistry, Eds. J. Demaison, J. E. Boggs, and A. G. Császár, (CRC Press, 2010, in print).

[17] H. Primas, Chemistry, Quantum Mechanics and Reductionism: Perspectives in Theoretical Chemistry (Springer-Verlag, Berlin, 1981), p. 13.

[18] A. Fröman and J. L. Kinsey, Phys. Rev. 123, 2077 (1961).

[19] A. K. Bhatia and A. Temkin, Phys. Rev. 137, A1335 (1965).

[20] J. M. Feagin and J. S. Briggs, Phys. Rev. Lett. 57, 984 (1986).

[21] J. M. Feagin and J. S. Briggs, Phys. Rev. A 37, 4599 (1988).

[22] A. M. Frolov, Phys. Rev. A 59, 4270 (1999).

[23] A. K. Bhatia and R. J. Drachman, Phys. Rev. A 59, 205 (1999).

[24] U. Müller-Herold, J. Chem. Phys 124, 014105 (2006).

[25] H. Primas, Acta Polytechnica Scandinavica MA 91, 83 (1998).

[26] G. Czakó, A. G. Császár, V. Szalay, and B. T. Sutcliffe J. Chem. Phys. 126, 024102 
(2007); C. Fábri, G. Czakó, G. Tasi, and A. G. Császár, J. Chem. Phys. 130, 134314 (2009).

[27] D. Luckhaus, J. Chem. Phys. 113, 1329 (2000).

[28] D. Lauvergnat and A. Nauts, J. Chem. Phys. 116, 8560 (2002).

[29] S. N. Yurchenko, W. Thiel, and P. Jensen, J. Mol. Spectrosc. 245, 126 (2007).

[30] E. Mátyus, G. Czakó, and A. G. Császár, J. Chem. Phys. 130, 134112 (2009).

[31] B. T. Sutcliffe, Molecular Hamiltonians, Handbook of Molecular Physics and Quantum Chemistry, Ed. Stephen Wilson, Volume 1, Part 6, Chapter 32, p. 501-525, John Wiley \& Sons, Ltd, Chichester (2003).

[32] Y. Suzuki and K. Varga, Stochastic Variational Approach to Quantum-Mechanical Few-Body Problems (Springer-Verlag, 1998).

[33] M. Cafiero, S. Bubin, and L. Adamowicz, Phys. Chem. Chem. Phys. 5, 1491 (2003).

[34] Y. Suzuki, J. Usukura, and K. Varga, J. Phys. B: At. Mol. Opt. Phys. 31, 31 (1998).

[35] M. Kamimura, Phys. Rev. A 38, 621 (1988).

[36] S. A. Alexander, H. J. Monkhorst, and K. Szalewicz, J. Chem. Phys. 85, 5821 (1986); ibid 87, 3976 (1987); ibid 89, 355 (1988).

[37] W. Kolos, C. C. J. Roothaan, and R. A. Sack, Rev. Mod. Phys. 32, 178 (1960).

[38] W. Kołos, and L. Wolniewicz, Rev. Mod. Phys. 35, 473 (1963).

[39] V. I. Korobov, Phys. Rev. A 61, 064503 (2000).

[40] G. W. F. Drake, M. M. Cassar, and R. A. Nistor, Phys. Rev. A 65, 054501 (2002).

[41] M. M. Cassar and G. W. F. Drake, J. Phys. B: At. Mol. Opt. Phys. 37, 2485 (2004).

[42] Y. Hijikata, H. Nakashima, and H. Nakatsuji, J. Chem. Phys. 130, 024102 (2009).

[43] M. Cafiero and L. Adamowicz, Chem. Phys. Lett. 387, 136 (2004).

[44] B. T. Sutcliffe and R. G. Woolley, Chem. Phys. Lett. 408, 445 (2005).

[45] M. Cafiero and L. Adamowicz, Int. J. Quant. Chem. 107, 2679 (2007).

[46] U. Müller-Herold, Eur. Phys. J. D 49, 311 (2008); U. Müller-Herold, Eur. Phys. J. D 56, 311 (2010).

[47] "2006 CODATA recommended values": http://physics.nist.gov/cuu/Constants/.

[48] T. Kato, Comm. Pure Appl. Math. 10, 151 (1957).

[49] S. Kais and Q. Shi, Phys. Rev. A 62, 060502(R) (2000); Q. Shi and S. Kais, Int. J. Quant. Chem. 85, 307 (2001). 
[50] R. F. W. Bader, Atoms in Molecules - A Quantum Theory (Oxford University Press, Oxford, 1990).

[51] J. Cioslowski and G. Liu, J. Chem. Phys. 110, 1882 (1999). 